\title{
Multifocal optical nanoscopy for big data recording at 30 TB capacity and gigabits/second data rate: supplementary material
}

\author{
XIANGPING LI ${ }^{1, \#}$, YAOYU CAO ${ }^{1, \#}$, NIAN TIAN ${ }^{2}$, LING FU², and MIN GU ${ }^{1, *}$ \\ ${ }^{1}$ Centre for Micro-Photonics, Faculty of Science, Engineering and Technology, Swinburne University of Technology, Hawthorn VIC 3122, Australia \\ ${ }^{2}$ Britton Chance Center for Biomedical Photonics, Wuhan National Laboratory for Optoelectronics-Huazhong University of Science and Technology, \\ Wuhan 430074, China \\ "These authors contributed equally to the work \\ *Corresponding author: mqu@swin.edu.au \\ Published 18 June 2015
}

This document provides supplementary information to "Multifocal optical nanoscopy for big data recording at $30 \mathrm{~TB}$ capacity and gigabits/second data rate," http://dx.doi.org/10.1364/optica.2.000567. We describe the phase retrieval method for in-plane and three-dimensional multifocal arrays, details of our experiments, and characterization of multifocal nanoscopy. (C) 2015 Optical Society of America

http://dx.doi.org/10.1364/optica.2.000567.s001

\section{ACCURATE PHASE MODULATION FOR IN-PLANE MULTIFOCAL ARRAYS}

In contrast to the scalar diffraction, the three orthogonal field components $\boldsymbol{E}_{\boldsymbol{x}}, \boldsymbol{E}_{\boldsymbol{y}}, \boldsymbol{E}_{\boldsymbol{z}}$, are calculated separately by the vectorial Debye integral [1,2]. Thus the overall intensity of the $\boldsymbol{k}$ th focal spot in the array equals to the modular square of the three components, given by

$$
I^{k}=\left|E_{x}^{k}\right|^{2}+\left|E_{y}^{k}\right|^{2}+\left|E_{z}^{k}\right|^{2}
$$

To enable uniform and diffraction-limited feature, an individual controlling factor $\boldsymbol{M}_{\boldsymbol{n}}^{\boldsymbol{k}}$ is introduced to correct the intensity of each of the focal spot in the multifocal array [3]:

$$
M_{n}^{k}=M_{n-1}^{k} \frac{I_{d}^{k}}{I_{n}^{k}}
$$

where $\boldsymbol{I}_{\boldsymbol{d}}^{\boldsymbol{k}}$ is the normalized intensity of the $\boldsymbol{k}$ th focal spot in the design, $\boldsymbol{I}_{\boldsymbol{n}}^{\boldsymbol{k}}$ is the intensity of the $\boldsymbol{k}$ th focal spot in the $\boldsymbol{n}$ th loop normalized by the average intensity. A high uniformity $(\boldsymbol{u}>$ $99 \%$ ) of focal spots with the desired binary value of " 1 ", which is crucial in the super-resolution recording, can be achieved through the iteration process [3]:

$$
u=1-\frac{I_{\max }-I_{\min }}{I_{\max }+I_{\min }}
$$

where $\boldsymbol{I}_{\boldsymbol{m a x}}$ and $\boldsymbol{I}_{\boldsymbol{m i n}}$ are the maximum and minimum intensities of the foci with the desired binary value of " 1 " in the array.

Fig. S1 shows the examples of retrieved phase patterns for 2 by 2 multifocal arrays for the induction and inhibition beam, respectively.

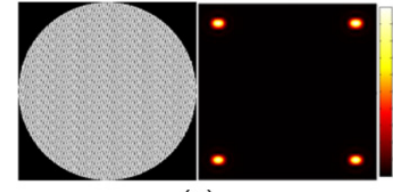

(a)

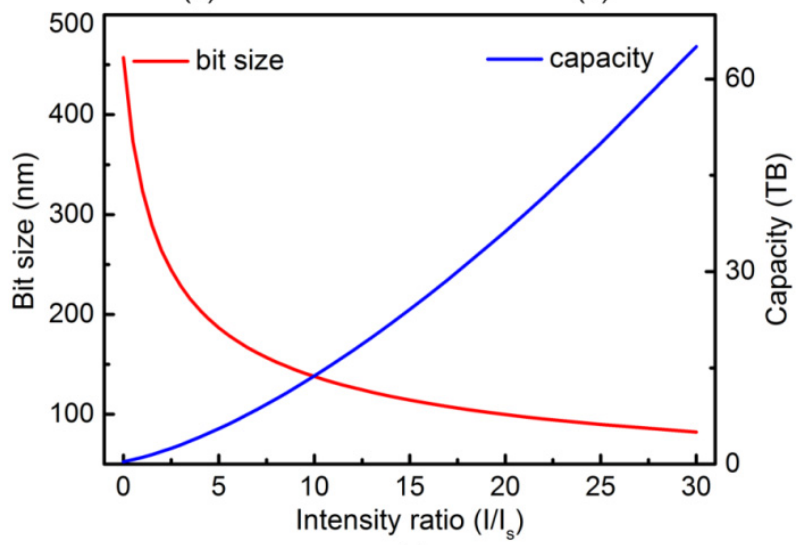

(c)

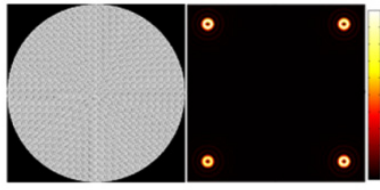

(b)
Fig S1. (a) The accurate phase modulation of the induction beam displayed on the SLM and the calculated 2 by 2 multifocal array in the focal plane. (b) The accurate phase modulation of the inhibition beam displayed on the SLM and the calculated 2 by 2 multifocal array with a doughnut-shaped focal spot. (c) The prediction of the recorded bit size as well as the equivalent capacity by increasing the inhibition intensity over the saturation intensity of the optical material. 


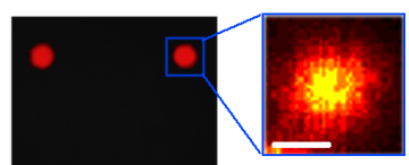

(a)

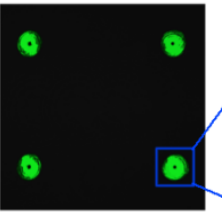

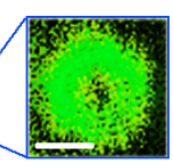

(b)

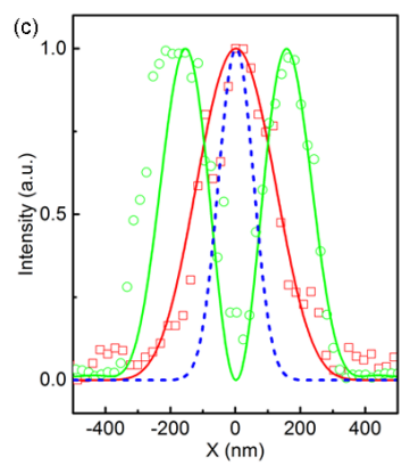

Fig. S2. CCD acquired images of the 2 by 2 multifocal array of the induction (a) and inhibition (b) beams. The insets show the characterization of the point spread function of each focal spot in the array by the raster scanning of fluorescent quantum dots. (c) The cross section plots of point spread functions of the induction and inhibition beams confirm the diffraction limited features and the spatial overlapping.

Fig. S2 shows the characterization of the point spread functions of multifocal arrays of the induction and inhibition beams, respectively, through the fluorescence imaging of single CdSe quantum dots. A photopolymerizable medium was prepared consisting of polymerization agents of SR 444 monomers and photoinitiators. A femtosecond pulsed laser beam at the wavelength of $800 \mathrm{~nm}$ was employed as the induction beam, while a continuous wave laser beam at the wavelength of $532 \mathrm{~nm}$ was employed as the inhibition beam. A high NA objective $(\mathrm{NA}=1.4)$ was used to focus the two beams in the focal plane. The scanning electron microscopic (SEM) images of feature sizes of the recorded bits and the separation between neighboring bits, at a variety of inhibition powers are shown in Fig. S3 and plotted in Fig.S4, respectively.

(a)

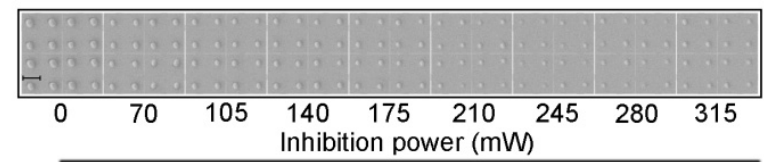

(b)

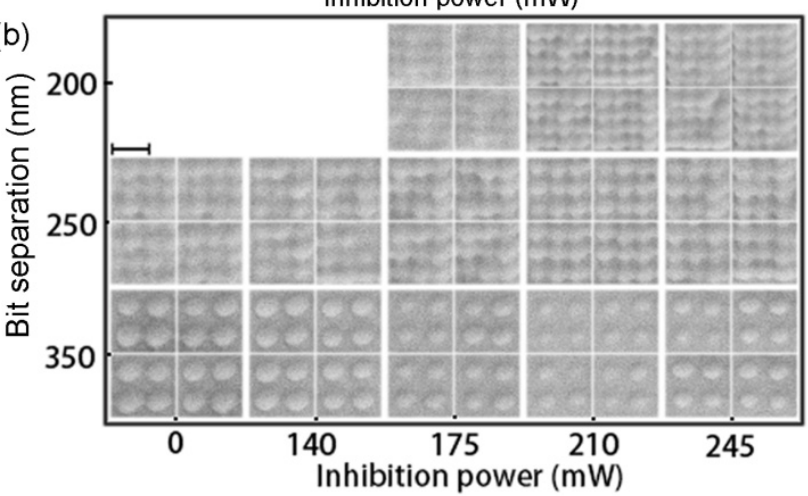

Fig. S3. SEM images of the feature size (a) and separation (b) of parallel recorded bits at a variety of the inhibition power of the multifocal array. Each subsection of the square is recorded by one of the focal spots in the 2 by 2 multifocal array. The scale bars are $500 \mathrm{~nm}$.

Another photopolymerizable sample consisting of 2,5-bis(pdimethylaminocinn amylidene)- cyclopentanone (BDMA), tetraethylthiuram disulfide (TED), SR 444 was prepared for high inhibition efficiency. Fig. S5 depicts the feature size as a function of the inhibition power in the new medium. The reduced inhibition power enables fast parallelism through 10 by 10 multifocal arrays, as shown in Fig. S6.

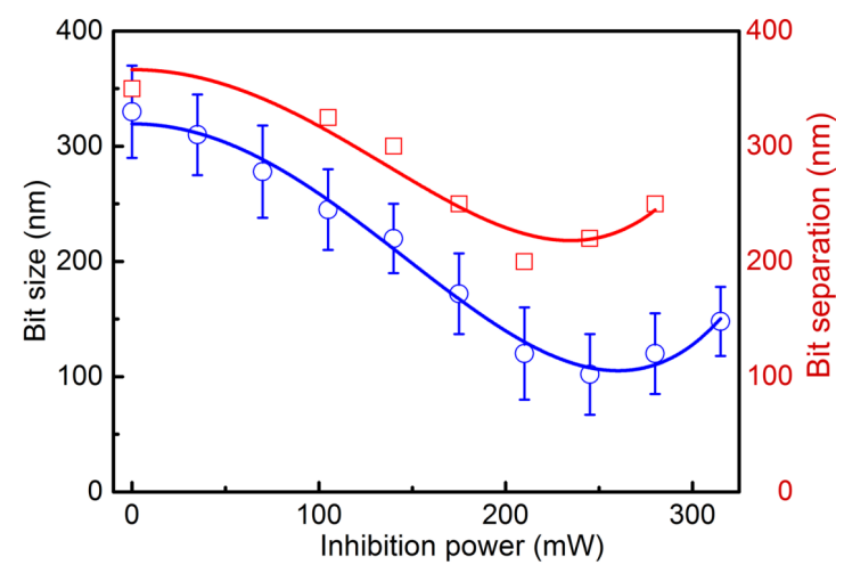

Fig. S4. The plot of the feature size (blue circles) and resolution (red squares) as a function of the inhibition power of the multifocal array. The power of the multifocal array of the induction beam is fixed at 36 $\mathrm{mW}$. The exposure time is $5 \mathrm{~ms}$.

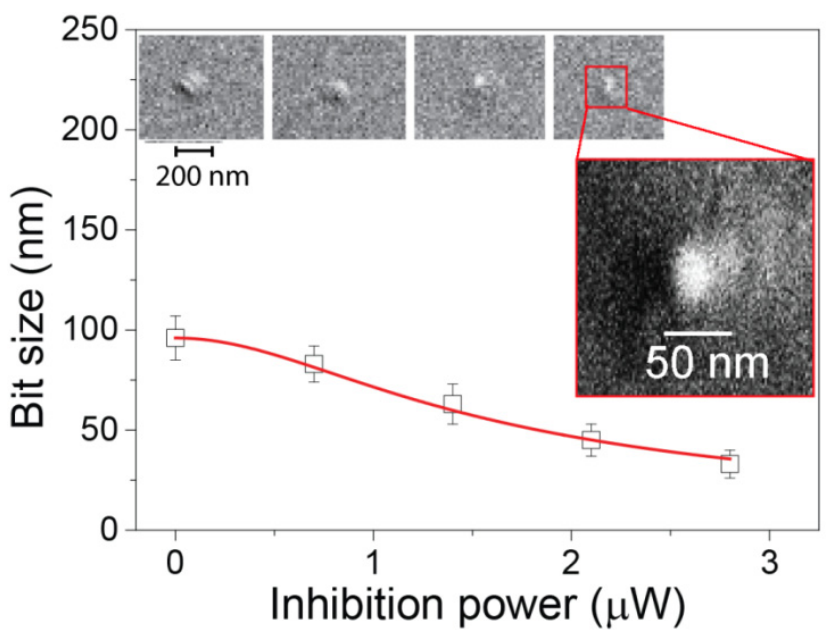

Fig. S5. Recorded bit size of the radical-quenching-based composites with the enhanced photoinhibition efficiency to the inhibition beam as a function of the inhibition power. The inset shows SEM images of the bit size obtained at various inhibition powers. The power of the induction beam is $8 \mathrm{~mW}$. The exposure time is $10 \mathrm{~ms}$. The fitting [5] gives the saturation power for the inhibition of $1.2 \mu \mathrm{W}$, corresponding to a three-order-of-magnitude reduction compared with that in the triplet-state-depletion-based photoresin.

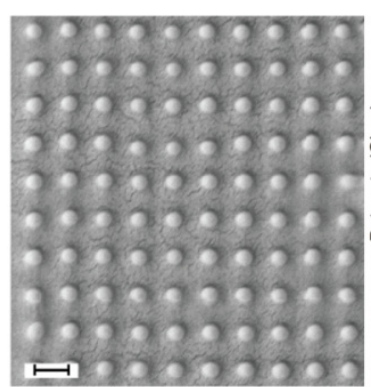

(a)

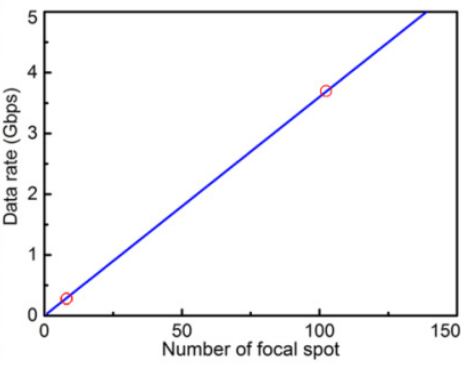

(b)
Fig. S6. (a) SEM image of the recorded pattern by a 10 by 10 superresolved multifocal array. The bit separation is $200 \mathrm{~nm}$. (b) The projected data rate by the parallelism through increasing the total number of focal spots in the multifocal array based on the data rate of Blu-ray at 36 Megabits/second. 


\section{ACCURATE PHASE MODULATION FOR THREE- DIMENSIONAL MULTIFOCAL ARRAYS}

In the case of three-dimensional (3D) parallel recording, a 3D entity field can be written as $\sum_{k=1}^{N} \boldsymbol{I}_{d}^{k} \boldsymbol{\delta}\left(\boldsymbol{x}_{k}, \boldsymbol{y}_{k}, \boldsymbol{z}_{k}\right)$, where $\boldsymbol{N}$ denotes the total foci number and $\boldsymbol{I}_{\boldsymbol{d}}^{\boldsymbol{k}}$ denotes the desired peak intensity of $\boldsymbol{k}$ th focal spot. The accurate phase is obtained through the vectorial Debye-based 3D-FT method on an Ewald cap and then projected to the back aperture plane [4].
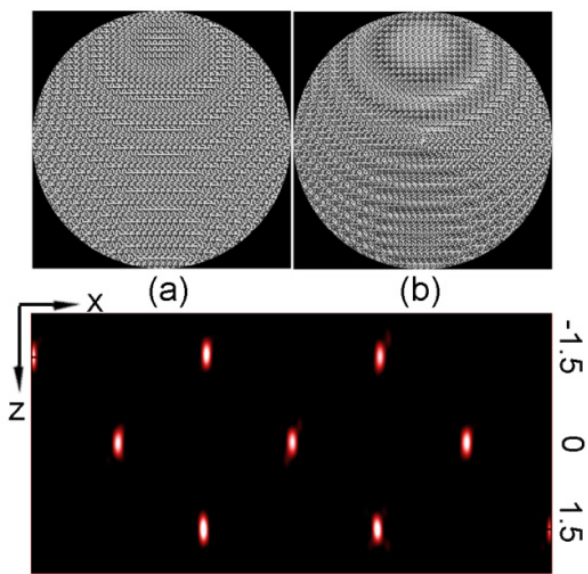

(c)

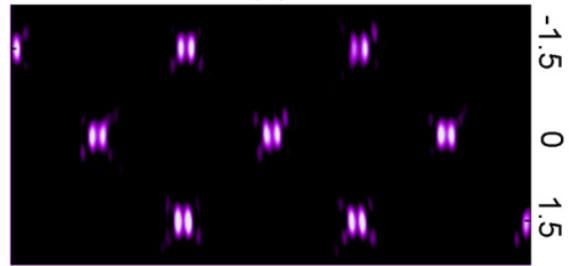

(d)

Fig. S7. The accurate phase modulations for the volumetric multifocal arrays for the induction beam (a) and inhibition beam (b). The multifocal array consists of three layers with 6 by 6 focal spots in each layer. A section of the axial plot of the calculated intensity distribution of the multifocal array for the induction beam (c) and the inhibition beam (d) with a layer separation of $1.5 \mu \mathrm{m}$.

Examples of retrieved phase modulations of volumetric multifocal arrays for the induction and inhibition beams, as well as the calculated axial intensity distribution of such threedimensional focal array are shown in Fig. S7.

\section{References}

1. B. Richards, and E. Wolf, "Electromagnetic diffraction in optical systems Il structure of the image field in an aplanatic system," Proc. Royal Soc. London Ser. A: Math. Phys. Sci. 253, 358-379 (1959).

2. M. Gu, Advanced Optical Imaging Theory (Springer, Verlag, 2000).

3. H. Lin, B. Jia, and M. Gu, "Dynamic generation of Debye diffractionlimited multifocal arrays for direct laser printing nanofabrication," Opt. Lett. 36, 406-408 (2011).

4. H. Ren, H. Lin, X. Li, and M. Gu, "Three-dimensional parallel recording with a Debye diffraction-limited and aberration-free volumetric multifocal array," Opt. Lett. 39, 1621-1624 (2014).

5. Y. Cao, Z. Gan, B. Jia, R. A. Evans, and M. Gu, "High-photosensitive resin for super-resolution direct-laser-writing based on photoinhibited polymerization," Opt. Express 19, 19486-19494 (2011). 